\title{
Expressive Key-Policy Attribute-Based Encryption with Constant-Size Ciphertexts
}

\author{
Nuttapong Attrapadung ${ }^{1}$, Benoît Libert ${ }^{2, \star}$, and Elie de Panafieu ${ }^{3}$ \\ 1 Research Center for Information Security, AIST, Japan \\ n. attrapadung@aist.go.jp \\ 2 Université catholique de Louvain, ICTEAM - Crypto Group, Belgium \\ benoit.libert@uclouvain.be \\ 3 Ecole Normale Supérieure, Cachan, France
}

\begin{abstract}
Attribute-based encryption (ABE), as introduced by Sahai and Waters, allows for fine-grained access control on encrypted data. In its key-policy flavor, the primitive enables senders to encrypt messages under a set of attributes and private keys are associated with access structures that specify which ciphertexts the key holder will be allowed to decrypt. In most ABE systems, the ciphertext size grows linearly with the number of ciphertext attributes and the only known exceptions only support restricted forms of threshold access policies.

This paper proposes the first key-policy attribute-based encryption (KP-ABE) schemes allowing for non-monotonic access structures (i.e., that may contain negated attributes) and with constant ciphertext size. Towards achieving this goal, we first show that a certain class of identitybased broadcast encryption schemes generically yields monotonic KPABE systems in the selective set model. We then describe a new efficient identity-based revocation mechanism that, when combined with a particular instantiation of our general monotonic construction, gives rise to the first truly expressive KP-ABE realization with constant-size ciphertexts. The downside of these new constructions is that private keys have quadratic size in the number of attributes. On the other hand, they reduce the number of pairing evaluations to a constant, which appears to be a unique feature among expressive KP-ABE schemes.
\end{abstract}

Keywords: Attribute-based encryption, expressivity, efficiency.

\section{Introduction}

It frequently happens that sensitive data must be archived by storage servers in such a way that only specific parties are allowed to read the content. In these situations, enforcing the access control using ordinary public key encryption schemes is not very convenient as such primitives severely decrease the flexibility of users to share their data.

\footnotetext{
* This author acknowledges the Belgian National Fund for Scientific Research (F.R.S.F.N.R.S.) for his "chargé de recherches" fellowship and the BCRYPT Interuniversity Attraction Pole.
}

D. Catalano et al. (Eds.): PKC 2011, LNCS 6571, pp. 90-108, 2011.

(C) International Association for Cryptologic Research 2011 
To address these concerns, Sahai and Waters 29] introduced attribute-based encryption (ABE), which refines identity-based encryption 30,8] by associating ciphertexts and private keys with sets of descriptive attributes. Decryption is then possible when there is a sufficient overlap between the two sets. These results were extended by Goyal, Pandey, Sahai and Waters [20] into richer kinds of attribute-based encryption, where decryption is permitted when the attribute set satisfies a more complex boolean formula specified by an access structure. This paper describes truly expressive ABE systems featuring compact ciphertexts, regardless of the number of underlying attributes.

Related Work. Attribute-based encryption comes in two flavors. In key-policy ABE schemes (KP-ABE), attribute sets are used to annotate ciphertexts and private keys are associated with access structures that specify which ciphertexts the user will be entitled to decrypt. Ciphertext-policy ABE (CP-ABE) proceeds in the dual way, by assigning attribute sets to private keys and letting senders specify an access policy that receivers' attribute sets should comply with.

The ciphertext-policy scenario was first studied in [5, 18. The construction of [18] only handles AND gates while the first expressive construction [5] was only analyzed in the generic group model. Goyal, Jain, Pandey and Sahai [21] gave a construction in the standard model but its large parameters and key sizes make it impractical for reasonably expressive policies. Efficient and expressive realizations in the standard model were subsequently put forth by Waters 32 . and one of them was recently extended by Lewko et al. [25], and subsequently by Okamoto and Takashima 31, into schemes providing adaptive security whereas all prior works on ABE were limited to deal with selective adversaries [13, 14, 6] - who have to make up their mind about their target before having seen public parameters - in their security analysis.

In both $\mathrm{CP}-\mathrm{ABE}$ and $\mathrm{KP}-\mathrm{ABE}$ schemes, expressivity requires to go beyond what monotonic access structures can express. Ostrovsky, Sahai and Waters [28] considered access structures that may contain negative attributes without blowing up the size of shares or ciphertexts. Their initial construction was recently improved by Lewko, Sahai and Waters 24] who used techniques from revocation systems (which can be seen as negative analogues of identity-based broadcast encryption) to design the most efficient non-monotonic KP-ABE to date.

Our Contributions. So far, the research community has mostly focused on the design of expressive schemes - where access structures can implement as complex boolean formulas as possible - without trying to minimize the size of ciphertexts. Indeed, most schemes [20, 28, 32, 25, 24] feature linear-size ciphertexts in the maximal number of attributes that ciphertexts can be annotated with. In the ciphertext-policy setting, Emura et al. suggested a scheme with short ciphertexts [19] but policies are restricted to a single AND gate. More recently, Herranz et al. 22] described a scheme with threshold access policies and constant-siz 11 ciphertexts. Yet, their scheme is still not as expressive as one

${ }^{1}$ By "constant", we mean that the size only depends on the security parameter $\lambda$ (the number of transmitted bits is typically $O(\lambda)$ ) and not on the number of ciphertext attributes. 
could hope for. In particular, it seems difficult to extend it to support general linear-secret-sharing-realizable (or LSSS-realizable for short) access structures.

In the context of key-policy attribute-based encryption, this paper aims at devising schemes with constant-size ciphertext:2 (regardless of the number of ciphertext attributes) allowing for as expressive policies as possible. To this end, we first show that a certain class of identity-based broadcast encryption (IBBE) schemes readily yields KP-ABE schemes with monotonic (though LSSSrealizable) access structures via a generic transformation. The latter preserves the ciphertext size and guarantees the resulting scheme to be selectively secure (as defined in [13,6]) as long as the underlying IBBE system is itself selectively secure. At the expense of quadratic-size private keys (which comprise $O(t \cdot n)$ elements, where $n$ is the maximal number of ciphertext attributes and $t$ is the maximal number of leaf attributes in access trees), this transformation directly provides us with monotonic KP-ABE schemes with $O(1)$-size ciphertexts.

In a second step, we use a particular output of the aforementioned transformation to design a scheme supporting non-monotonic access structures without sacrificing the efficiency. In the resulting construction, the ciphertext overhead reduces to three group elements, no matter how many attributes ciphertexts are associated with. As in the monotonic case, private keys are inflated by a factor of $n$ in comparison with [28,24]. Nevertheless, these new schemes remain attractive for applications where bandwidth is the primary concern. In mobile Internet connections for instance, users are charged depending on the amount of transmitted messages; while in contrast, the storage is becoming much cheaper nowadays even for a large amount, as evidently in many smart phones.

As an intermediate step towards the new non-monotonic ABE, we design a new identity-based revocation (IBR) mechanism (as defined by Lewko, Sahai and Waters [24]) with $O(1)$-size ciphertexts and a similar structure to that of the monotonic KP-ABE schemes provided by our general construction. This was necessary since prior IBR systems with short ciphertexts [4 were not directly amenable to fulfill these requirements. We believe this new IBR realization to be of independent interest since it performs noticeably better than previous schemes featuring short ciphertexts [4 and still relies a natural (though " $q$-type") intractability assumption.

The security of our schemes is proved against selective adversaries (that are not allowed to choose their target attribute set adaptively) under a noninteractive assumption. We leave it as an open problem to obtain KP-ABE schemes with compact ciphertexts that can be proven secure against adaptive adversaries (as in the work of Lewko et al. 25]).

Other Related Work. The aforementioned realizations all assume ABE schemes with a single authority and we focus on this context as well. Extensions to the multi-authority scenario were investigated in [15, 16] for a conjunctive

\footnotetext{
${ }^{2}$ As in the literature on broadcast encryption (see, e.g., 9]) where the list of receivers is not included in the ciphertext, we do not count the description of ciphertext attributes as being part of the ciphertext. Indeed, many ciphertexts may have to be encrypted under the same attribute set.
} 
setting and in [3] for a disjunctive setting. Besides the two usual flavors of ABE, another recently considered kind of ABE schemes [2], called dual-policy ABE, mixes features from both $\mathrm{KP}-\mathrm{ABE}$ and $\mathrm{CP}-\mathrm{ABE}$ systems.

OrganizAtion. In the following, we first review various primitives in section 2 , Section 3 describes our general construction of monotonic KP-ABE. The new revocation scheme is depicted in section 4 . Section 5 finally presents the nonmonotonic ABE realization with compact ciphertexts.

\section{Background and Definitions}

Notation. We will treat a vector as a column vector, unless stated otherwise. Namely, for any vector $\boldsymbol{\alpha}=\left(\alpha_{1}, \ldots, \alpha_{n}\right)^{\top} \in \mathbb{Z}_{p}^{n}, g^{\boldsymbol{\alpha}}$ stands for the vector of group elements $\left(g^{\alpha_{1}}, \ldots, g^{\alpha_{n}}\right)^{\top} \in \mathbb{G}^{n}$. For $\boldsymbol{a}, \boldsymbol{z} \in \mathbb{Z}_{p}^{n}$, we denote their inner product as $\langle\boldsymbol{a}, \boldsymbol{z}\rangle=\boldsymbol{a}^{\top} \boldsymbol{z}=\sum_{i=1}^{n} a_{i} z_{i}$. Given $g^{\boldsymbol{a}}$ and $\boldsymbol{z},\left(g^{\boldsymbol{a}}\right)^{\boldsymbol{z}}:=g^{\langle\boldsymbol{a}, \boldsymbol{z}\rangle}$ is computable without knowing $\boldsymbol{a}$. We denote by $I_{n}$ the identity matrix of size $n$. For a set $U$, we define $2^{U}=\{S \mid S \subseteq U\}$ and $\left(\begin{array}{c}U \\ <k\end{array}\right)=\{S|S \subseteq U| S \mid,<k\}$ for $k \leq|U|$.

\subsection{Syntax and Security Definition for Functional Encryption}

We capture notions of KP-ABE, IBBE, IBR by providing a unified definition and security notion for functional encryption 3 here and then instantiating to these primitives in the next subsections.

Syntax. Let $R: \Sigma_{k} \times \Sigma_{e} \rightarrow\{0,1\}$ be a boolean function where $\Sigma_{k}$ and $\Sigma_{e}$ denote "key index" and "ciphertext index" spaces. A functional encryption (FE) scheme for the relation $R$ consists of algorithms: Setup, KeyGen, Encrypt, Decrypt.

$\operatorname{Setup}(\lambda, \operatorname{des}) \rightarrow($ mpk, msk): The setup algorithm takes as input a security parameter $\lambda$ and a scheme description des and outputs a master public key mpk and a master secret key msk.

$\operatorname{KeyGen}(\mathrm{msk}, X) \rightarrow \mathrm{sk}_{X}$ : The key generation algorithm takes in the master secret key msk and a key index $X \in \Sigma_{k}$. It outputs a private key $\mathrm{sk}_{X}$.

Encrypt (mpk, M, $Y) \rightarrow C$ : This algorithm takes as input a public key mpk, the message $\mathrm{M}$, and a ciphertext index $Y \in \Sigma_{e}$. It outputs a ciphertext $C$.

$\operatorname{Decrypt}\left(\mathrm{mpk}, \mathrm{sk}_{X}, X, C, Y\right) \rightarrow \mathrm{M}$ or $\perp$ : The decryption algorithm takes in the public parameters mpk, a private key $\mathrm{sk}_{X}$ for the key index $X$ and a ciphertext $C$ for the ciphertext index $Y$. It outputs the message $\mathrm{M}$ or a symbol $\perp$ indicating that the ciphertext is not in a valid form.

Correctness mandates that, for all $\lambda$, all (mpk, msk) produced by $\operatorname{Setup}(\lambda$, des),

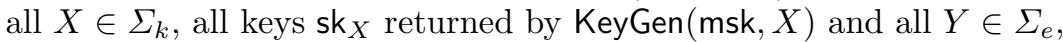

- If $R(X, Y)=1$, then Decrypt(mpk, Encrypt(mpk, M, $Y))$, sk $\left.{ }_{X}\right)=\mathrm{M}$.

- If $R(X, Y)=0$, then Decrypt(mpk, Encrypt(mpk, M, $Y))$, sk $\left._{X}\right)=\perp$.

${ }^{3}$ The term "functional encryption" was defined in slightly different manners in 25,4, 31. before recently fully formalized in [11. Our definition of FE here and throughout the paper refers to the class of predicate encryption with public index of [1]. 
Security Notion. We now give the standard security definition for FE schemes.

Definition 1. A FE scheme for relation $R$ is fully secure if no probabilistic polynomial time (PPT) adversary $\mathcal{A}$ has non-negligible advantage in this game:

Setup. The challenger runs $(\mathrm{mpk}, \mathrm{msk}) \leftarrow \operatorname{Setup}(\lambda$, des $)$ and gives mpk to $\mathcal{A}$.

Phase 1. On polynomially-many occasions, $\mathcal{A}$ chooses a key index $X$ and gets $\mathrm{sk}_{X}=\operatorname{Keygen}(\mathrm{msk}, X)$. Such queries can be adaptive in that each one may depend on the information gathered so far.

Challenge. $\mathcal{A}$ chooses messages $\mathrm{M}_{0}, \mathrm{M}_{1}$ and a ciphertext index $Y^{\star}$ such that $R\left(X, Y^{\star}\right)=0$ for all key indexes $X$ that have been queried at step 2. Then, the challenger flips a fair binary coin $d \in\{0,1\}$, generates a ciphertext $C^{\star}=\operatorname{Encrypt}\left(\mathrm{mpk}, \mathrm{M}_{d}, Y^{\star}\right)$, and hands it to the adversary.

Phase 2. $\mathcal{A}$ is allowed to make more key generation queries for any key index $X$ such that $R\left(X, Y^{\star}\right)=0$.

Guess. $\mathcal{A}$ outputs a bit $d^{\prime} \in\{0,1\}$ and wins if $d^{\prime}=d$.

The advantage of the adversary $\mathcal{A}$ is measured by $\operatorname{Adv}(\lambda):=\left|\operatorname{Pr}\left[d^{\prime}=d\right]-\frac{1}{2}\right|$.

A weaker notion called selective security [13, 6] can be defined as in the above game with the exception that the adversary $\mathcal{A}$ has to choose the challenge ciphertext index $Y^{\star}$ before the setup phase but private key queries $X_{1}, \ldots, X_{q}$ can still be adaptive. A dual notion called co-selective security 4, in contrast, requires $\mathcal{A}$ to declare $q$ key queries for key indexes $X_{1}, \ldots, X_{q}$ before the setup phase, but $\mathcal{A}$ can adaptively choose the target challenge ciphertext index $Y^{\star}$.

\subsection{Key-Policy Attribute-Based Encryption}

Before describing the definition of $\mathrm{KP}-\mathrm{ABE}$, we first recall the definitions of access structures and linear secret sharing schemes, as defined in [20].

Definition 2 (Access Structures). Consider a set of parties $\mathcal{P}=\left\{P_{1}, P_{2}, \ldots\right.$, $\left.P_{n}\right\}$. A collection $\mathbb{A} \subseteq 2^{\mathcal{P}}$ is said to be monotone if, for all $B, C$, if $B \in \mathbb{A}$ and $B \subseteq C$, then $C \in \mathbb{A}$. An access structure (resp., monotonic access structure) is a collection (resp., monotone collection) $\mathbb{A} \subseteq 2^{\mathcal{P}} \backslash\{\emptyset\}$. The sets in $\mathbb{A}$ are called the authorized sets, and the sets not in $\mathbb{A}$ are called the unauthorized sets.

Definition 3 (Linear Secret Sharing Scheme). Let $\mathcal{P}$ be a set of parties. Let $L$ be a $\ell \times k$ matrix. Let $\pi:\{1, \ldots, \ell\} \rightarrow \mathcal{P}$ be a function that maps a row to a party for labeling. A secret sharing scheme $\Pi$ for access structure $\mathbb{A}$ over a set of parties $\mathcal{P}$ is a linear secret-sharing scheme (LSSS) in $\mathbb{Z}_{p}$ and is represented by $(L, \pi)$ if it consists of two efficient algorithms:

Share $_{(L, \pi)}$ : takes as input $s \in \mathbb{Z}_{p}$ which is to be shared. It chooses $\beta_{2}, \ldots, \beta_{k} \stackrel{R}{\leftarrow}$ $\mathbb{Z}_{p}$ and let $\boldsymbol{\beta}=\left(s, \beta_{2}, \ldots, \beta_{k}\right)^{\top}$. It outputs $L \cdot \boldsymbol{\beta}$ as the vector of $\ell$ shares. The share $\lambda_{i}:=\left\langle\boldsymbol{L}_{\boldsymbol{i}}, \boldsymbol{\beta}\right\rangle$ belongs to party $\pi(i)$, where $\boldsymbol{L}_{\boldsymbol{i}}{ }^{\top}$ is the $i^{\text {th }}$ row of $L$. $\operatorname{Recon}_{(L, \pi)}$ : takes as input an access set $S \in \mathbb{A}$. Let $I=\{i \mid \pi(i) \in S\}$. It outputs a set of constants $\left\{\left(i, \mu_{i}\right)\right\}_{i \in I}$ such that $\sum_{i \in I} \mu_{i} \cdot \lambda_{i}=s$. 
In a key-policy attribute-based encryption scheme, ciphertexts are associated with a set of attributes $\omega$ and private keys correspond to access structures $\mathbb{A}$. Decryption is possible when the attribute set $\omega$ is authorized in the access structure $\mathbb{A}$ (i.e., $\omega \in \mathbb{A}$ ). We formally define it as an instance of $\mathrm{FE}$ as follows.

Definition 4 (KP-ABE). Let $U$ be an attribute space. Let $n \in \mathbb{N}$ be a bound on the number of attributes per ciphertext. A key-policy attribute-based encryption (KP-ABE) for a collection $\mathcal{A S}$ of access structures over $U$ is a functional encryption for $R^{\mathrm{KP}}: \mathcal{A S} \times\left(\begin{array}{c}U \\ <n\end{array}\right) \rightarrow\{0,1\}$ defined by $R^{\mathrm{KP}}(\mathbb{A}, \omega)=1$ iff $\omega \in \mathbb{A}$ (for $\omega \subseteq U$ such that $|\omega|<n$, and $\mathbb{A} \in \mathcal{A S}$ ). Furthermore, the description des consists of the attribute universe $U, \Sigma_{k}^{\mathrm{KP}}=\mathcal{A S}$, and $\Sigma_{e}^{\mathrm{KP}}=\left(\begin{array}{c}U \\ <n\end{array}\right)$.

Definition 4 conforms with the original definition of KP-ABE, as in 20, 28, 24, 25. 11. There is another variant of KP-ABE recently used in 31, that we call $\mathrm{KP}-\mathrm{ABE}$ with labeling. We re-formalize it in appendix A, for the purpose of comparison in Table 2. We remark that normal KP-ABE implies KP-ABE with labeling.

We note that chosen-ciphertext secure versions of our proposed KP-ABE schemes in this paper can be obtained from recent generic results of [33].

\subsection{Identity-Based Broadcast Encryption and Revocation Scheme}

An ID-based broadcast encryption, as formalized in [1], allows a sender to encrypt a message to a set of identities, say $S=\left\{\mathrm{ID}_{1}, \ldots, \mathrm{ID}_{q}\right\}$, where $q<n$ for some a-priori fixed bound $n \in \mathbb{N}$, so that a user who possesses a key for ID $\in S$ can decrypt. In contrast, an ID-based revocation scheme 24] allows a sender to specify a revoked set $S$ so that only a user with ID $\notin S$ can decrypt.

Definition 5. Let $\mathcal{I}$ be an identity space. An ID-based broadcast encryption scheme (IBBE) with the maximal bound $n$ for the number of receivers per ciphertext is a functional encryption for $R^{\mathrm{IBBE}}: \mathcal{I} \times\left(\begin{array}{c}\mathcal{I} \\ <n\end{array}\right) \rightarrow\{0,1\}$ defined by $R^{\mathrm{IBBE}}(\mathrm{ID}, S)=1$ iff ID $\in S$.

Definition 6. Let $\mathcal{I}$ be an identity space. An ID-based revocation (IBR) with the maximal bound $n$ for the number of revoked users per ciphertext is a functional encryption for $R^{\mathrm{IBR}}: \mathcal{I} \times\left(\begin{array}{c}\mathcal{I} \\ <n\end{array}\right) \rightarrow\{0,1\}$ defined by $R^{\mathrm{IBR}}(\mathrm{ID}, S)=1$ iff ID $\notin S$.

Remark 1. Although selective and co-selective security are incomparable in general, we remark that, in IBR schemes, co-selective security implies selective security. To see why, we first recall that selective security for IBR requires the adversary $\mathcal{A}$ to declare the target revoked set $S^{\star}$ before seeing the public key mpk. Here, phase 1 can be simplified by letting the challenger hand over all the private keys for identities in $S^{\star}$ at once (along with mpk). On the other hand, co-selective IBR security requires $\mathcal{A}$ to declare the set $\tilde{S}$ of identities that will be queried for private key generation before seeing mpk whereas the target revocation set $S^{\star}$ does not have to be fully determined before the challenge phase. At the same time as mpk, the challenger then reveals all keys for identities in $\tilde{S}$ at once. Later, the adversary can choose any $S^{\star} \subseteq \tilde{S}$ in the challenge phase. Selective security corresponds to the special case where $S^{\star}=\tilde{S}$. 


\subsection{Complexity Assumptions}

We use groups $\left(\mathbb{G}, \mathbb{G}_{T}\right)$ of prime order $p$ with an efficiently computable mapping $e: \mathbb{G} \times \mathbb{G} \rightarrow \mathbb{G}_{T}$ s.t. $e\left(g^{a}, h^{b}\right)=e(g, h)^{a b}$ for any $(g, h) \in \mathbb{G} \times \mathbb{G}, a, b \in \mathbb{Z}$ and $e(g, h) \neq 1_{\mathbb{G}_{T}}$ whenever $g, h \neq 1_{\mathbb{G}}$. We rely on the DBDHE assumption introduced in 9 . This assumption is shown to hold in the generic group model 7 . In addition, it is non-interactive and falsifiable [26].

Definition 7. In $\left(\mathbb{G}, \mathbb{G}_{T}\right)$, the $q$-Decision Bilinear Diffie-Hellman Exponent $(q-D B D H E)$ problem is, given $\left(g, g^{\gamma}, g^{\left(\gamma^{2}\right)}, \ldots, g^{\left(\gamma^{q}\right)}, g^{\left(\gamma^{q+2}\right)}, \ldots, g^{\left(\gamma^{2 q}\right)}, h, T\right)$ where $\gamma \stackrel{R}{\leftarrow} \mathbb{Z}_{p}, g, h \stackrel{R}{\leftarrow} \mathbb{G}$ and $T \in_{R} \mathbb{G}_{T}$, to decide if $T=e(g, h)^{\left(\gamma^{q+1}\right)}$ or if $T$ is a random element of $\mathbb{G}_{T}$.

\section{Monotonic KP-ABE with Short Ciphertexts}

Our first goal is to construct monotonic KP-ABE with short ciphertexts. We do so by showing a general transformation that automatically turns any IBBE scheme fitting a certain template into a KP-ABE in the selective security model.

The construction is somewhat similar to the one described by Boyen [12, which transforms IBE in the exponent-inversion framework to ABE. The approach of 12 took advantage of certain linearity properties in a family of IBE schemes. Our approach also exploits some linearity properties, albeit instead of IBE, we use IBBE as the underlying primitive. In contrast to [12, our transformation preserves the ciphertext size, hence using IBBE with short ciphertexts will yield KP-ABE with the same ciphertext size.

\subsection{Linear ID-Based Broadcast Encryption Template}

We define a template that IBBE schemes should comply with in order to give rise to (selectively secure) KP-ABE schemes. We call this a linear IBBE template. Let $\left(\mathbb{G}, \mathbb{G}_{T}\right)$ be underlying bilinear groups of order $p$. A linear IBBE scheme is determined by parameter $n_{1}, n_{2} \in \mathbb{N}$, a family $\mathcal{F}$ of vectors of functions, and a function $\mathcal{D}$, of which the latter two are specified by

$$
\begin{aligned}
& \mathcal{F} \subset\left\{\left(f_{1}, f_{2}, F\right) \mid f_{1}: \mathbb{Z}_{p}^{*} \rightarrow \mathbb{G}, f_{2}: \mathbb{Z}_{p}^{*} \rightarrow \mathbb{G}^{n_{1}}, F:\left(\mathbb{Z}_{p}^{*}\right)^{\leq n-1} \rightarrow \mathbb{G}^{\leq n_{2}}\right\}, \\
& \mathcal{D}: \mathbb{G}^{n_{1}+2} \times \mathcal{I} \times \mathbb{G}^{\leq n_{2}+1} \times\left(\begin{array}{c}
\mathcal{I} \\
<n
\end{array}\right) \rightarrow \mathbb{G}_{T},
\end{aligned}
$$

with requirements specified below. A linear IBBE scheme works as follows.

- Setup $(\lambda, n)$ : Given a security parameter $\lambda \in \mathbb{N}$ and a bound $n \in \mathbb{N}$ on the number of identities per ciphertext, the algorithm selects bilinear groups $\left(\mathbb{G}, \mathbb{G}_{T}\right)$ of prime order $p$ and a generators $g \stackrel{R}{\leftarrow} \mathbb{G}$. It computes $e(g, g)^{\alpha}$ for a random $\alpha \stackrel{R}{\longleftarrow} \mathbb{Z}_{p}^{*}$ and chooses functions $\left(f_{1}, f_{2}, F\right) \stackrel{R}{\longleftarrow} \mathcal{F}$. The master secret key consists of msk $:=g^{\alpha}$ while the public key is mpk $:=\left(g, e(g, g)^{\alpha}, f_{1}, f_{2}, F, n, n_{1}, n_{2}\right)$.

- Keygen (msk, ID): It picks $r \stackrel{R}{\leftarrow} \mathbb{Z}_{p}^{*}$ and computes

$$
\mathbf{s k}_{\mathrm{ID}}=\left(d_{1}, d_{2}, d_{3}\right)=\left(g^{\alpha} \cdot f_{1}(\mathrm{ID})^{r}, g^{r}, f_{2}(\mathrm{ID})^{r}\right) \in \mathbb{G}^{n_{1}+2} .
$$


- Encrypt (mpk, M, $S)$ : It parses $S$ as $S=\left\{\mathrm{ID}_{1}, \ldots, \mathrm{ID}_{q}\right\}$, where $q<n$. To encrypt $\mathrm{M} \in \mathbb{G}_{T}$, it chooses a random exponent $s \stackrel{R}{\leftarrow} \mathbb{Z}_{p}^{*}$ and computes the ciphertext as

$$
C=\left(C_{0}, C_{1}, C_{2}\right)=\left(\mathrm{M} \cdot e(g, g)^{\alpha \cdot s}, g^{s}, F\left(\mathrm{ID}_{1}, \ldots, \mathrm{ID}_{q}\right)^{s}\right) .
$$

$\operatorname{Decrypt}\left(\mathrm{mpk}, \mathrm{sk}_{\mathrm{ID}}, \mathrm{ID}, C, S\right)$ : It parses sk $\mathrm{ID}_{\mathrm{D}}=\left(d_{1}, d_{2}, d_{3}\right)$ and $C=\left(C_{0}, C_{1}, C_{2}\right)$ then runs

$$
\mathcal{D}\left(\left(d_{1}, d_{2}, d_{3}\right), \mathrm{ID},\left(C_{1}, C_{2}\right), S\right) \rightarrow e(g, g)^{\alpha \cdot s},
$$

and obtains $\mathrm{M}=C_{0} / e(g, g)^{\alpha \cdot s}$. We are now ready to state the requirements: for all $\left(f_{1}, f_{2}, F\right) \in \mathcal{F}$, the following two properties must hold.

1. Correctness. For all $\alpha, r, s \in \mathbb{Z}_{p}^{*}$, ID $\in \mathcal{I}, S=\left\{\mathrm{ID}_{1}, \ldots, \mathrm{ID}_{q}\right\} \in\left(\begin{array}{c}\mathcal{I} \\ <n\end{array}\right)$ and $\mathrm{ID} \in S$, we have

$$
\mathcal{D}\left(\left(g^{\alpha} f_{1}(\mathrm{ID})^{r}, g^{r}, f_{2}(\mathrm{ID})^{r}\right), \mathrm{ID},\left(g^{s}, F\left(\mathrm{ID}_{1}, \ldots, \mathrm{ID}_{q}\right)^{s}\right), S\right)=e(g, g)^{\alpha \cdot s} .
$$

2. Linearity. For all $\gamma \in \mathbb{Z}_{p}^{*}$, ID $\in \mathcal{I}, S \in\left(\begin{array}{c}\mathcal{I} \\ <n\end{array}\right), \mathrm{ID} \in S,\left(d_{1}, d_{2}, d_{3}\right) \in \mathbb{G}^{n_{1}+2}$, and $\left(C_{1}, C_{2}\right) \in \mathbb{G}^{\leq n_{2}+1}$, we have

$$
\mathcal{D}\left(\left(d_{1}, d_{2}, d_{3}\right)^{\gamma}, \mathrm{ID},\left(C_{1}, C_{2}\right), S\right)=\mathcal{D}\left(\left(d_{1}, d_{2}, d_{3}\right), \mathrm{ID},\left(C_{1}, C_{2}\right), S\right)^{\gamma} .
$$

\subsection{Generic Conversion from Linear IBBE to KP-ABE}

Let $\Pi_{\mathrm{IBBE}}=\left(\right.$ Setup $^{\prime}$, Keygen $^{\prime}$, Encrypt $^{\prime}$, Decrypt $\left.^{\prime}\right)$ be a linear IBBE system. We construct a KP-ABE scheme from $\Pi_{\mathrm{IBBE}}$ as follows.

- Setup $(\lambda, n)$ : It simply outputs $\operatorname{Setup}^{\prime}(\lambda, n) \rightarrow$ (msk, mpk).

- Keygen $(\mathrm{msk},(L, \pi))$ : The algorithm computes a private key for an access structure that is associated with LSSS scheme $(L, \pi)$ as follows. Let $L$ be $\ell \times k$ matrix. First, it generates shares of 1 with the $\operatorname{LSSS}(L, \pi)$. Namely, it chooses a vector $\boldsymbol{\beta}=\left(\beta_{1}, \beta_{2}, \ldots, \beta_{k}\right)^{\top} \stackrel{R}{\leftarrow}\left(\mathbb{Z}_{p}\right)^{k}$ subject to the constraint $\beta_{1}=1$. Then for each $i=1$ to $\ell$, it calculates $\lambda_{i}=\left\langle\boldsymbol{L}_{\boldsymbol{i}}, \boldsymbol{\beta}\right\rangle$, picks $r^{\prime} \stackrel{R}{\leftarrow} \mathbb{Z}_{p}$ and sets $D_{i}$ as follows.

$$
\begin{aligned}
\text { Keygen }^{\prime}(\mathrm{msk}, \pi(i)) & \rightarrow\left(d_{i, 1}, d_{i, 2}, d_{i, 3}\right), \\
D_{i} & =\left(d_{i, 1}^{\lambda_{i}} \cdot f_{1}(\pi(i))^{r^{\prime}}, d_{i, 2}^{\lambda_{i}} \cdot g^{r^{\prime}}, d_{i, 3}^{\lambda_{i}} \cdot f_{2}(\pi(i))^{r^{\prime}}\right) .
\end{aligned}
$$

It then outputs the private key as $\operatorname{sk}_{(L, \pi)}=\left\{D_{i}\right\}_{i=1, \ldots, \ell}$.

- Encrypt (mpk, M, $\omega)$ : It simply outputs Encrypt $(m p k, \mathrm{M}, \omega) \rightarrow\left(C_{0}, C_{1}, C_{2}\right)$.

- Decrypt $\left(\mathrm{mpk}, \mathrm{sk}_{(L, \pi)},(L, \pi), C, \omega\right)$ : Assume first that the policy $(L, \pi)$ is satisfied by the attribute set $\omega$, so that decryption is possible. Let $I=\{i \mid \pi(i) \in \omega\}$. It calculates the reconstruction constants $\left\{\left(i, \mu_{i}\right)\right\}_{i \in I}=\operatorname{Recon}_{(L, \pi)}(\omega)$. It parses $C$ as $\left(C_{0}, C_{1}, C_{2}\right)$ and sk $_{(L, \pi)}$ as $\left\{D_{i}\right\}_{i=1, \ldots, \ell}$ where $D_{i}=\left(d_{i, 1}^{\prime}, d_{i, 2}^{\prime}, d_{i, 3}^{\prime}\right)$. For each $i \in I$, it computes

$$
\mathcal{D}\left(\left(d_{i, 1}^{\prime}, d_{i, 2}^{\prime}, d_{i, 3}^{\prime}\right), \mathrm{ID},\left(C_{1}, C_{2}\right), S\right) \rightarrow e(g, g)^{\alpha \cdot s \cdot \lambda_{i}},
$$

which we prove correctness below. It computes $e(g, g)^{\alpha \cdot s}=\prod_{i \in I}\left(e(g, g)^{\alpha \cdot s \cdot \lambda_{i}}\right)^{\mu_{i}}$ and finally obtains $\mathrm{M}=C_{0} / e(g, g)^{\alpha \cdot s}$, where we recall that $\sum_{i \in I} \lambda_{i} \mu_{i}=1$. 
Correctness. We now verify that equation (11) is correct. First from a property of keys in linear IBBE, we have that $\left(d_{i, 1}, d_{i, 2}, d_{i, 3}\right)$ will be in the form $\left(g^{\alpha}\right.$. $\left.f_{1}(\pi(i))^{r_{i}}, g^{r_{i}}, f_{2}(\pi(i))^{r_{i}}\right)$ for some $r_{i} \in_{R} \mathbb{Z}_{p}$. Therefore, we have

$$
D_{i}=\left(g^{\alpha \lambda_{i}} \cdot f_{1}(\pi(i))^{\tilde{r}_{i} \lambda_{i}}, g^{\tilde{r}_{i} \lambda_{i}}, f_{2}(\pi(i))^{\tilde{r}_{i} \lambda_{i}}\right)=\left(d_{1}^{\lambda_{i}}, d_{2}^{\lambda_{i}}, d_{3}^{\lambda_{i}}\right),
$$

with $\tilde{r}_{i}=r_{i}+r^{\prime} / \lambda_{i}$ and $\left(d_{1}, d_{2}, d_{3}\right)=$ sk $_{\pi(i)}$ with randomness $\tilde{r}_{i}$. Hence,

$$
\begin{aligned}
\mathcal{D}\left(\left(d_{i, 1}^{\prime}, d_{i, 2}^{\prime}, d_{i, 3}^{\prime}\right), \mathrm{ID},\left(C_{1}, C_{2}\right), S\right) & =\mathcal{D}\left(\left(d_{1}, d_{2}, d_{3}\right), \mathrm{ID},\left(C_{1}, C_{2}\right), S\right)^{\lambda_{i}} \\
& =\left(e(g, g)^{\alpha \cdot s}\right)^{\lambda_{i}},
\end{aligned}
$$

where each equality holds from linearity and correctness of $\mathcal{D}$ respectively.

The construction only guarantees selective security for the resulting KP-ABE. It does not extend to the adaptive scenario because the proof relies on the fact that the reduction knows the forbidden attribute set from the beginning.

Theorem 1. If the underlying IBBE scheme is selectively secure, then the resulting KP-ABE system is also selectively secure. (The proof is given in the full version of the paper).

Instantiation ExAmPLE. The large-universe construction of KP-ABE in 20] falls into our framework here. Its underlying IBBE system can be seen as a particular instance of the linear IBBE template with $n_{2}=n, f_{2}(\mathrm{ID})=\emptyset$, $F\left(\mathrm{ID}_{1}, \ldots, \mathrm{ID}_{q}\right)=\left(f_{1}\left(\mathrm{ID}_{1}\right), \ldots, f_{1}\left(\mathrm{ID}_{q}\right)\right)$, and the form of $f_{1}$ can be straightforwardly deduced from [20]. Since the size of an output from $F$ is linear, ciphertexts in the KP-ABE of 20] are also of linear size.

\subsection{IBBE Instantiation with Short Ciphertexts}

This subsection presents an IBBE scheme with short ciphertexts and shows how to apply the KP-ABE conversion. This specific IBBE can be seen as an instance of the functional encryption (FE) for zero inner-product proposed in [4, Sect.4.1], which itself is implied by spatial encryption of [10]. A FE system for zero innerproduct is defined by a relation $R^{\mathrm{ZIP}}: \mathbb{Z}_{p} \times \mathbb{Z}_{p} \rightarrow\{0,1\}$ where $R^{\mathrm{ZIP}}(\boldsymbol{X}, \boldsymbol{Y})=1$ iff $\langle\boldsymbol{X}, \boldsymbol{Y}\rangle=0$. The technique of deriving an IBBE scheme from a $\mathrm{FE}$ scheme for zero inner-product can be traced to [23]. A private key for an identity ID is defined by setting $\boldsymbol{X}=\left(x_{1}, \ldots, x_{n}\right)^{\top}$, with $x_{i}=\mathrm{ID}^{i-1}$. To encrypt to a set $S=\left\{\mathrm{ID}_{1}, \ldots, \mathrm{ID}_{q}\right\}$, one defines $\boldsymbol{Y}=\left(y_{1}, \ldots, y_{n}\right)^{\top}$ as a coefficient vector from

$$
P_{S}[Z]=\sum_{i=1}^{q+1} y_{i} Z^{i-1}=\prod_{\mathbb{I D}_{j} \in S}\left(Z-\mathrm{ID}_{j}\right),
$$

where, if $q+1<n$, the coordinates $y_{q+2}, \ldots, y_{n}$ are set to 0 . By doing so, we note that $P_{S}[\mathrm{ID}]=\langle\boldsymbol{X}, \boldsymbol{Y}\rangle$ evaluates to 0 iff ID $\in S$. We now describe the IBBE instantiated from the FE system of [4]. Its selective security is an immediate consequence of [4, where it is proved under the DBDHE assumption. 
- Setup $(\lambda, n)$ : It chooses bilinear groups $\left(\mathbb{G}, \mathbb{G}_{T}\right)$ of prime order $p>2^{\lambda}$ with $g \stackrel{R}{\leftarrow} \mathbb{G}$. It randomly chooses $\alpha, \alpha_{0} \stackrel{R}{\leftarrow} \mathbb{Z}_{p}, \boldsymbol{\alpha}=\left(\alpha_{1}, \ldots, \alpha_{n}\right)^{\top} \stackrel{R}{\leftarrow} \mathbb{Z}_{p}^{n}$. It then sets $\boldsymbol{H}=\left(h_{1}, \ldots, h_{n}\right)^{\top}=g^{\boldsymbol{\alpha}}$. The master secret key is msk $=\alpha$, and the public key is mpk $=\left(g, e(g, g)^{\alpha}, h_{0}=g^{\alpha_{0}}, \boldsymbol{H}=g^{\boldsymbol{\alpha}}\right)$.

- Keygen(msk, ID): The algorithm first defines a vector $\boldsymbol{X}=\left(x_{1}, \ldots, x_{n}\right)^{\top}$ such that $x_{i}=\mathrm{ID}^{i-1}$ for $i=1$ to $n$. It chooses $r \stackrel{R}{\leftarrow} \mathbb{Z}_{p}$ and outputs the private key as $\mathbf{s k}_{\mathrm{ID}}=\left(D_{1}, D_{2}, K_{2}, \ldots, K_{n}\right)$ where

$$
D_{1}=g^{\alpha} \cdot h_{0}^{r}, \quad D_{2}=g^{r}, \quad\left\{K_{i}=\left(h_{1}^{-\frac{x_{i}}{x_{1}}} \cdot h_{i}\right)^{r}\right\}_{i=2, \ldots, n} .
$$

- Encrypt $(\mathrm{mpk}, \mathrm{M}, S)$ : To encrypt $\mathrm{M}$ to the receiver set $S$ (where $|S|<n$ ), the algorithm defines $\boldsymbol{Y}=\left(y_{1}, \ldots, y_{n}\right)^{\top}$ as the coefficient vector of $P_{S}[Z]$ from equation (21). It then picks $s \stackrel{R}{\longleftarrow} \mathbb{Z}_{p}$ and computes the ciphertext as

$$
C=\left(C_{0}, C_{1}, C_{2}\right)=\left(\mathrm{M} \cdot e(g, g)^{\alpha s}, g^{s},\left(h_{0} \cdot h_{1}^{y_{1}} \cdots h_{n}^{y_{n}}\right)^{s}\right) .
$$

- Decrypt $\left(\mathrm{mpk}, \mathrm{sk}_{\mathrm{ID}}, \mathrm{ID}, C, S\right)$ : It defines the vector $\boldsymbol{Y}=\left(y_{1}, \ldots, y_{n}\right)^{\top}$ from the polynomial $P_{S}[Z]$ as usual. It then computes

$$
e(g, g)^{\alpha \cdot s}=\frac{e\left(C_{1}, D_{1} \cdot K_{2}^{y_{2}} \cdots K_{n}^{y_{n}}\right)}{e\left(C_{2}, D_{2}\right)},
$$

and recovers $\mathrm{M}=C_{0} / e(g, g)^{\alpha \cdot s}$.

CorRectness. If $\langle\boldsymbol{X}, \boldsymbol{Y}\rangle=0$, then decryption recovers $\mathrm{M}$ since

$$
D_{1} \cdot \prod_{i=2}^{n} K_{i}^{y_{i}}=g^{\alpha} \cdot\left(h_{0} \cdot h_{1}^{-\frac{1}{x_{1}}\left(\langle\boldsymbol{X}, \boldsymbol{Y}\rangle-x_{1} y_{1}\right)} \prod_{i=2}^{n} h_{i}^{y_{i}}\right)^{r}=g^{\alpha} \cdot\left(h_{0} \cdot \prod_{i=1}^{n} h_{i}^{y_{i}}\right)^{r},
$$

so that $e\left(C_{1}, D_{1} \cdot \prod_{i=1}^{n} K_{i}^{y_{i}}\right)=e(g, g)^{\alpha s} \cdot e\left(h_{0} \cdot \prod_{i=1}^{n} h_{i}^{y_{i}}, g^{r s}\right)$ equals the product $e(g, g)^{\alpha s} \cdot e\left(C_{2}, D_{2}\right)$.

Applying the KP-ABE Conversion. The above IBBE can be considered as a linear IBBE system with $n_{1}=n-1, n_{2}=1$ and the family $\mathcal{F}$ is defined by taking all functions of the following forms ranging over $h_{0}, h_{1}, \ldots, h_{n} \in \mathbb{G}$ :

$$
f_{1}(\mathrm{ID})=h_{0}, f_{2}(\mathrm{ID})=\left(h_{1}^{-\mathrm{ID}} h_{2}, \ldots, h_{1}^{-\mathrm{ID}^{n-1}} h_{n}\right), F\left(\mathrm{ID}_{1}, \ldots, \mathrm{ID}_{q}\right)=h_{0} \prod_{i=1}^{q+1} h_{i}^{y_{i}},
$$

where the vector $\boldsymbol{Y}=\left(y_{1}, \ldots, y_{n}\right)^{\top}$ is defined from the polynomial $P_{S}[Z]$ in equation (2) as usual. In addition, the function $\mathcal{D}$ is the computation in equation (3), which can be shown to have linearity as required.

The resulting KP-ABE has constant-size ciphertexts. This comes with the expense of longer private keys of size $O(t \cdot n)$, where $t$ is the number of attributes in the access structure. It is also worth mentioning that we can obtain another IBBE with short ciphertexts from the spatial encryption scheme of [10 since it also falls into our framework and thus produces another KP-ABE scheme.

Our goal in this paper is to construct KP-ABE with non-monotonic structures. We will combine the monotonic KP-ABE system in this subsection with new IDbased revocation in the next section. 


\section{Revocation Scheme with Very Short Ciphertexts}

This section describes a new ID-based revocation system which is tailored to the needs of our application. Analogously to the case of IBBE, an IBR scheme can be instantiated from a FE system for non-zero inner-product relations. Two such existing IBR schemes 4, Sect. 5.1 and 5.2] already provide constant-size ciphertexts. When it comes to construct a non-monotonic KP-ABE however, these schemes seem hardly compatible with the monotonic KP-ABE of section 3.3 as they rely on different assumptions. We thus describe a new IBR scheme for this purpose. Its structure is similar to that of revocation schemes given in [4] but it provides a better efficiency and relies on the DBDHE assumption.

- Setup $(\lambda, n)$ : It chooses bilinear groups $\left(\mathbb{G}, \mathbb{G}_{T}\right)$ of prime order $p>2^{\lambda}$ and a generator $g \stackrel{R}{\leftarrow} \mathbb{G}$. It randomly picks $\alpha \stackrel{R}{\longleftarrow} \mathbb{Z}_{p}, \boldsymbol{\alpha}=\left(\alpha_{1}, \ldots, \alpha_{n}\right)^{\top} \stackrel{R}{\longleftarrow} \mathbb{Z}_{p}^{n}$ and sets $\boldsymbol{H}=\left(h_{1}, \ldots, h_{n}\right)^{\top}=g^{\boldsymbol{\alpha}}$. The master secret key is msk $=\alpha$, while the public key is mpk $=\left(g, e(g, g)^{\alpha}, \boldsymbol{H}=g^{\boldsymbol{\alpha}}\right)$.

- Keygen(msk, ID): The algorithm first defines a vector $\boldsymbol{X}=\left(x_{1}, \ldots, x_{n}\right)^{\top}$ such that $x_{i}=\mathrm{ID}^{i-1}$ for $i=1$ to $n$. It chooses $r \stackrel{R}{\leftarrow} \mathbb{Z}_{p}$ and outputs the private key as $\mathrm{sk}_{\mathrm{ID}}=\left(D_{1}, D_{2}, K_{2}, \ldots, K_{n}\right)$ where

$$
D_{1}=g^{\alpha} \cdot h_{1}^{r}, \quad D_{2}=g^{r}, \quad\left\{K_{i}=\left(h_{1}^{-\frac{x_{i}}{x_{1}}} \cdot h_{i}\right)^{r}\right\}_{i=2, \ldots, n} .
$$

Indeed, we can also write $K_{\boldsymbol{X}}=\left(K_{2}, \ldots, K_{n}\right)=g^{r \cdot M_{\boldsymbol{X}}^{\top} \boldsymbol{\alpha}}$, where the matrix $M_{\boldsymbol{X}} \in\left(\mathbb{Z}_{p}\right)^{n \times(n-1)}$ is defined by $M_{\boldsymbol{X}}=\left(\begin{array}{c}-\frac{x_{2}}{x_{1}}-\frac{x_{3}}{x_{1}} \cdots-\frac{x_{n}}{x_{1}} \\ I_{n-1}\end{array}\right)$.

- Encrypt(mpk, M,S): To encrypt $\mathrm{M}$ with the revoked set $S$ (where $|S|<n$ ), the algorithm defines $\boldsymbol{Y}=\left(y_{1}, \ldots, y_{n}\right)^{\top}$ as the coefficient vector of $P_{S}[Z]$ from equation (2). It then picks $s \stackrel{R}{\leftarrow} \mathbb{Z}_{p}$ and computes the ciphertext as

$$
C=\left(C_{0}, C_{1}, C_{2}\right)=\left(\mathrm{M} \cdot e(g, g)^{\alpha \cdot s}, g^{s},\left(h_{1}^{y_{1}} \cdots h_{n}^{y_{n}}\right)^{s}\right) .
$$

- Decrypt(mpk, sk ID ID, $C, S)$ : It defines $\boldsymbol{X}$ from ID and $\boldsymbol{Y}$ from $S$ as usual. It then successively computes elements $K=\prod_{i=2}^{n} K_{i}^{y_{i}}=\left(h_{1}^{-\langle\boldsymbol{X}, \boldsymbol{Y}\rangle / x_{1}} \cdot h_{1}^{y_{1}} \cdots h_{n}^{y_{n}}\right)^{r}$, $\tau=\left(\frac{e\left(K, C_{1}\right)}{e\left(C_{2}, D_{2}\right)}\right)^{-\frac{x_{1}}{\langle\boldsymbol{X}, \boldsymbol{Y}\rangle}}=e\left(g, h_{1}\right)^{r s}$, and then obtains $\mathrm{M}=C_{0} \cdot e\left(C_{1}, D_{1}\right)^{-1} \cdot \tau$.

Correctness. We first observe that

$$
K=\left(h_{1}^{-\left(\langle\boldsymbol{X}, \boldsymbol{Y}\rangle-x_{1} y_{1}\right) / x_{1}} \prod_{i=2}^{n} h_{i}^{y_{i}}\right)^{r}=\left(h_{1}^{-\langle\boldsymbol{X}, \boldsymbol{Y}\rangle / x_{1}} \prod_{i=1}^{n} h_{i}^{y_{i}}\right)^{r}
$$

so that whenever $\langle\boldsymbol{X}, \boldsymbol{Y}\rangle \neq 0$ (i.e., ID $\notin S$ ), the following computation can be done.

$$
\tau=\left(\frac{e\left(K, C_{1}\right)}{e\left(C_{2}, D_{2}\right)}\right)^{-\frac{x_{1}}{\langle\boldsymbol{X}, \boldsymbol{Y}\rangle}}=\left(\frac{e\left(h_{1}^{-\langle\boldsymbol{X}, \boldsymbol{Y}\rangle / x_{1}} \prod_{i=1}^{n} h_{i}^{y_{i}}, g^{r s}\right)}{e\left(\prod_{i=1}^{n} h_{i}^{y_{i}}, g^{r s}\right)}\right)^{-\frac{x_{1}}{\langle\boldsymbol{X}, \boldsymbol{Y}\rangle}}=e\left(g, h_{1}\right)^{r s} .
$$


Finally, we have $e\left(C_{1}, D_{1}\right) \cdot \tau^{-1}=e(g, g)^{\alpha \cdot s} \cdot e\left(g^{s}, h_{1}^{r}\right) \cdot e\left(g, h_{1}\right)^{-r s}=e(g, g)^{\alpha \cdot s}$. We note that the decryption algorithm can be optimized by computing the plaintext as $\mathrm{M}=C_{0} \cdot e\left(C_{2}, D_{2}^{x_{1} /\langle\boldsymbol{X}, \boldsymbol{Y}\rangle}\right) \cdot e\left(C_{1}, D_{1}^{-1} \cdot K^{-x_{1} /\langle\boldsymbol{X}, \boldsymbol{Y}\rangle}\right)$.

At a high level, it shares the same structure (including the form of the public key and the ciphertext) as the IBBE in section 3.3 and relies on the same assumption. Intuitively, these similarities make it possible to assemble both constructions in the design of a non-monotonic ABE system in section 5 ,

We now prove the co-selective security of the scheme. It is also worth recalling that co-selective security for IBR also implies selective security.

Theorem 2. The above ID-based revocation scheme with the maximal bound $n$ for the number of revoked users (i.e., $|S|<n$ ) is co-selectively secure if the $n$-DBDHE assumption holds in $\left(\mathbb{G}, \mathbb{G}_{T}\right)$.

Proof. We show an algorithm $\mathcal{B}$ that receives $\left(g, h, z_{1}, \ldots, z_{n}, z_{n+2}, \ldots, z_{2 n}, T\right)$ in $\mathbb{G}^{2 n+1} \times \mathbb{G}_{T}$, where $z_{i}=g^{\left(\gamma^{i}\right)}$, and decides if $T=e(g, h)^{\left(\gamma^{n+1}\right)}$ using the co-selective adversary $\mathcal{A}$.

At the outset of the game, the adversary $\mathcal{A}$ declares the set $\tilde{S}=\left\{\mathrm{ID}_{1}, \ldots, \mathrm{ID}_{q}\right\}$, where $q \leq n-1$, of identities for which she wishes to obtain private keys. Let $\boldsymbol{X}_{1}, \ldots, \boldsymbol{X}_{q}$ the corresponding vectors. That is, $\boldsymbol{X}_{k}=\left(1, \mathrm{ID}_{k}, \mathrm{ID}_{k}^{2}, \ldots, \mathrm{ID}_{k}^{n-1}\right)$. To prepare the public key, $\mathcal{B}$ chooses $\delta_{0} \stackrel{R}{\leftarrow} \mathbb{Z}_{p}$ and computes $e(g, g)^{\alpha}=e\left(z_{1}, z_{n}\right)^{\delta_{0}}$, which implicitly defines $\alpha=\gamma^{(n+1)} \cdot \delta_{0}$. Elements $\boldsymbol{H}=\left(h_{1}, \ldots, h_{n}\right)^{\top}$ are then defined as follows. For each $k \in[1, q], \mathcal{B}$ considers the vector $\boldsymbol{X}_{k}=\left(x_{k, 1}, \ldots, x_{k, n}\right)^{\top}$ and selects $\boldsymbol{b}_{k} \in \mathbb{Z}_{p}^{n}$ such that

$$
\boldsymbol{b}_{k}^{\top} \cdot M_{\boldsymbol{X}_{\boldsymbol{k}}}=\boldsymbol{b}_{k}^{\top} \cdot\left(\begin{array}{c}
-\frac{x_{k, 2}}{x_{k, 1}}-\frac{x_{k, 3}}{x_{k, 1}} \cdots-\frac{x_{k, n}}{x_{k, 1}} \\
I_{n-1}
\end{array}\right)=\mathbf{0} .
$$

The simplest candidate consists of the vector $\boldsymbol{b}_{k}=\left(1, \frac{x_{k, 2}}{x_{k, 1}}, \frac{x_{k, 3}}{x_{k, 1}}, \ldots, \frac{x_{k, n}}{x_{k, 1}}\right)^{\top}$. Then, $\mathcal{B}$ considers the $n \times n$ matrix $B=\left(\boldsymbol{b}_{1}|\ldots| \boldsymbol{b}_{q}|\mathbf{0}| \ldots \mid \mathbf{0}\right)$ whose $k^{\text {th }}$ column consists of $\boldsymbol{b}_{k}$, for $k=1$ to $q$, and where the $n-q$ remaining columns are 0. It defines $\boldsymbol{a}=\left(a_{1}, \ldots, a_{n}\right)^{\top} \in\left(\mathbb{Z}_{p}\right)^{n}$ such that $a_{i}=\gamma^{n+1-i}$ by setting $g^{\boldsymbol{a}}=\left(z_{n}, \ldots, z_{1}\right)^{\top}$. Then, it implicitly sets $\boldsymbol{\alpha}=B \cdot \boldsymbol{a}+\boldsymbol{\delta}$ by randomly choosing $\boldsymbol{\delta} \stackrel{R}{\leftarrow} \mathbb{Z}_{p}^{n}$ and defining $\boldsymbol{H}=g^{B \cdot \boldsymbol{a}} \cdot g^{\boldsymbol{\delta}}$, which is uniformly distributed as required.

Due to (4), the matrix $B$ is defined in such a way that, for each $k \in[1, q]$, the $k^{\text {th }}$ column of $M_{\boldsymbol{X}_{k}}^{\top} \cdot B \in\left(\mathbb{Z}_{p}\right)^{(n-1) \times n}$ is $\mathbf{0}$, so that $M_{\boldsymbol{X}_{k}}^{\top} \cdot B \cdot \boldsymbol{a}$ does not contain $a_{k}=\gamma^{n+1-k}$. Then, a private key for the identity $\operatorname{ID}_{k}$ (and thus the vector $\boldsymbol{X}_{k}$ ) can be obtained by implicity defining $\tilde{r}_{k}=r_{k}-\delta_{0} \gamma^{k}$ for a random $r_{k} \stackrel{R}{\leftarrow} \mathbb{Z}_{p}$. Indeed, with the above choice of $B$, the first coordinate of $\boldsymbol{\alpha}=\boldsymbol{\delta}+\sum_{j=1}^{q} a_{j} \boldsymbol{b}_{j}$ equals $\alpha_{1}=\delta_{1}+\sum_{j=1}^{q} a_{j}=\delta_{1}+\sum_{j=1}^{q} \gamma^{(n+1-j)}$, so that $\mathcal{B}$ is able to compute

$$
\begin{aligned}
D_{1}=g^{\alpha} \cdot h_{1}^{\tilde{r}_{k}} & =g^{\left(\gamma^{n+1}\right) \delta_{0}} \cdot h_{1}^{r_{k}} \cdot\left(g^{\delta_{1}} \cdot \prod_{j=1}^{q} z_{n+1-j}\right)^{-\delta_{0} \gamma_{k}} \\
& =h_{1}^{r_{k}} \cdot\left(z_{k}^{\delta_{1}} \cdot \prod_{j=1, j \neq k}^{q} z_{n+1-j+k}\right)^{-\delta_{0}}
\end{aligned}
$$


and $D_{2}=g^{r_{k}} \cdot z_{n+1-k}^{-\delta_{0}}$. As for the delegation component $K_{\boldsymbol{X}_{k}}=g^{\tilde{r}_{k} M_{\boldsymbol{X}_{k}}^{\top} \boldsymbol{\alpha}}, \mathcal{B}$ is also able to compute it from available values since $M_{\boldsymbol{X}_{k}}^{\top} \boldsymbol{\alpha}=M_{\boldsymbol{X}_{k}}^{\top} \cdot B \cdot \boldsymbol{a}+M_{\boldsymbol{X}_{k}}^{\top} \cdot \boldsymbol{\delta}$ is independent of $a_{k}=\gamma^{n+1-k}$ (recall that the $k^{\text {th }}$ column of $M_{\boldsymbol{X}_{k}}^{\top} \cdot B$ is $\mathbf{0}$ ) and no term $\gamma^{n+1}$ appears in the exponent in $K_{\boldsymbol{X}_{k}}$.

In the challenge phase, $\mathcal{B}$ chooses $\mathrm{M}_{0}, \mathrm{M}_{1} \in \mathbb{G}_{T}$ and a revocation set $S$ corresponding to a vector $\boldsymbol{Y}=\left(y_{1}, \ldots, y_{n}\right)^{\top}$ that must satisfy $\left\langle\boldsymbol{X}_{k}, \boldsymbol{Y}\right\rangle=0$ for $k=1$ to $q$. This amounts to say that $\boldsymbol{Y}=M_{\boldsymbol{X}_{k}} \cdot \boldsymbol{w}$, where $\boldsymbol{w}=\left(y_{2}, \ldots, y_{n}\right)^{\top}$, for each $k \in[1, q]$. We claim that $\boldsymbol{Y}^{\top} \cdot B \cdot \boldsymbol{a}=0$. Indeed,

$$
\boldsymbol{Y}^{\top} \cdot B \cdot \boldsymbol{a}=\boldsymbol{Y}^{\top} \cdot\left(\sum_{k=1}^{q} a_{k} \cdot \boldsymbol{b}_{k}\right)=\sum_{k=1}^{q} a_{k} \cdot \boldsymbol{Y}^{\top} \cdot \boldsymbol{b}_{k}=\sum_{k=1}^{q} a_{k} \cdot \boldsymbol{w}^{\top} \cdot M_{\boldsymbol{X}_{k}}^{\top} \cdot \boldsymbol{b}_{k}
$$

and $M_{\boldsymbol{X}_{k}}^{\top} \cdot \boldsymbol{b}_{k}=\mathbf{0}$ for each $k \in[1, q]$. Therefore, it comes that $\langle\boldsymbol{Y}, \boldsymbol{\alpha}\rangle=\langle\boldsymbol{Y}, \boldsymbol{\delta}\rangle$, so that $\mathcal{B}$ can generate a challenge ciphertext $\left(C_{0}, C_{1}, C_{2}\right)$ as

$$
C_{0}=\mathrm{M}_{d} \cdot T^{\delta_{0}}, \quad C_{1}=h, \quad C_{2}=h^{\langle\boldsymbol{Y}, \boldsymbol{\delta}\rangle},
$$

for a random bit $d \stackrel{R}{\leftarrow}\{0,1\}$. If $T=e(g, h)^{\left(\gamma^{n+1}\right)}, C=\left(C_{0}, C_{1}, C_{2}\right)$ forms a valid encryption of $\mathrm{M}_{d}$. If $T$ is random, $\mathcal{A}$ 's advantage is clearly zero.

EFFICIENCY COMPARISONs. We believe this IBR scheme to be of interest in its own right. If we compare it with the scheme of [4, Sect.5.2] (called AL2 here), which also features short ciphertexts, it relies on a stronger assumption (since no " $q$-type" assumption is needed in [4] or in LSW2 24]) but provides significantly shorter ciphertexts (as the ciphertext overhead is decreased by more than 75\%) 14 and requires fewer pairing evaluations to decrypt (only 2 instead of 9 ). Another IBR scheme (dubbed AL1 in the table) with a better efficiency than AL2 was described in [4, Sect.5.1]. Still, the new scheme is slightly more efficient and relies on a weaker assumption since $q$-DBDHE is weaker and appears more natural than the $q$-type assumption (MEBDH) used in [24,4].

Table 1. Performances of revocation systems

\begin{tabular}{lccccc}
\hline Schemes & \multicolumn{6}{c}{$\begin{array}{c}\text { Ciphertext overhead Private key size Decryption cost } \\
\text { Assumption }\end{array}$} & $|\mathbb{G}|$ & pair. & exp. & \\
\hline LSW1 [24] & $|\mathbb{G}|$ & 3 & 3 & $O(\bar{n})$ & $n$-MEBDH \\
LSW2 [24] & $(2 \bar{n}+1)$ & 7 & 9 & $O(\bar{n})$ & DLIN, DBDH \\
AL1 [4] & 3 & $(n+2)$ & 3 & $O(n)$ & $n$-MEBDH \\
AL2 [4] & 9 & $(n+2)$ & 9 & $O(n)$ & DLIN, DBDH \\
This work & 2 & $(n+2)$ & 2 & $O(n)$ & $n$-DBDHE \\
\hline
\end{tabular}

${ }^{\dagger} \bar{n}=\#$ of revoked users $=|S| ; n=$ the maximal bound for $\bar{n}$. (i.e., $\left.|S|<n\right)$.

$\ddagger$ pair.,exp. shows \# of pairing and exponentiation computation.

${ }^{4}$ We compare by simple element counting. In a stricter sense, one may want to also consider the compensation due to the attack on $q$-type assumptions by Cheon [17. 
In comparison with the schemes of Lewko, Sahai and Waters, the disadvantage lies in that a bound on the number of revocations must be chosen when the system is set up. A comparative efficiency of known IBR schemes is given in the table hereafter.

\section{Non-monotonic KP-ABE with Short Ciphertexts}

Ostrovsky, Sahai and Waters 28] suggested a technique to move from monotonic to non-monotonic access structures without incurring an immoderate private key size. They assume a family $\left\{\Pi_{\mathbb{A}}\right\}_{\mathbb{A} \in \mathcal{A S}}$ of linear secret-sharing schemes for a set of monotone access structures $\mathbb{A}$. For each such access structure $\mathbb{A} \in \mathcal{A S}$, the set $\mathcal{P}$ of underlying parties is defined in such a way that parties' names can be normal (like $x$ ) or primed (like $x^{\prime}$ ). Prime attributes are conceptually seen as the negation of unprimed attributes. In addition, it is required that, if $x \in \mathcal{P}$, then $x^{\prime} \in \mathcal{P}$ and vice versa.

A family $\mathcal{A S}$ of non-monotone access structures can be defined as follows. For each access structure $\mathbb{A} \in \mathcal{A S}$ over a set of parties $\mathcal{P}$, one defines a possibly non-monotonic access structure $N M(\mathbb{A})$ over the set $\tilde{\mathcal{P}}$ of all unprimed parties in $\mathcal{P}$. An operator $N($.) is then defined as follows. For every set $\tilde{S} \subset \tilde{\mathcal{P}}$, one imposes $\tilde{S} \subset N(\tilde{S})$. Also, for each $x \in \tilde{\mathcal{P}}$ such that $x \notin \tilde{S}, x^{\prime} \in N(\tilde{S})$. Finally, $N M(\mathbb{A})$ is defined by saying that $\tilde{S}$ is authorized in $N M(\mathbb{A})$ if and only if $N(\tilde{S})$ is authorized in $\mathbb{A}$ (so that $N M(\mathbb{A})$ has only unprimed parties in its access sets). For each access set $X \in N M(\mathbb{A})$, there is a set in $\mathbb{A}$ containing the elements in $X$ and primed elements for each party not in $X$.

In [28, the above technique was combined with the Naor-Pinkas revocation method [27] to cope with non-monotonic access structures. Lewko, Sahai and Waters provided improvements using a revocation system with short keys [24] instead of [27]. In the following, we apply the same technique to our revocation mechanism and combine it with the monotonic KP-ABE derived from the IBBE scheme of section 3.3 in order to handle non-negated attributes.

- Setup $(\lambda, n)$ : Given a security parameter $\lambda \in \mathbb{N}$ and a bound $n \in \mathbb{N}$ of the number of attributes per ciphertext, it chooses bilinear groups $\left(\mathbb{G}, \mathbb{G}_{T}\right)$ of prime order $p>2^{\lambda}$ and $g \stackrel{R}{\leftarrow} \mathbb{G}$. It defines $\boldsymbol{H}=\left(h_{1}, \ldots, h_{n}\right)^{\top}$ and $\boldsymbol{U}=\left(u_{0}, \ldots, u_{n}\right)^{\top}$ such that $h_{i}=g^{\alpha_{i}}, u_{j}=g^{\beta_{j}}$ for each $i \in\{1, \ldots, n\}$ and $j \in\{0, \ldots, n\}$ where $\boldsymbol{\alpha}=\left(\alpha_{1}, \ldots, \alpha_{n}\right)^{\top} \stackrel{R}{\leftarrow} \mathbb{Z}_{p}^{n}$ and $\boldsymbol{\beta}=\left(\beta_{0}, \beta_{1}, \ldots, \beta_{n}\right)^{\top} \stackrel{R}{\leftarrow} \mathbb{Z}_{p}^{n+1}$. It then picks $\alpha \stackrel{R}{\leftarrow} \mathbb{Z}_{p}^{*}$ and computes $e(g, g)^{\alpha}$. The master secret key is msk $=\alpha$ and the master public key is mpk $=\left(g, e(g, g)^{\alpha}, \boldsymbol{H}=g^{\boldsymbol{\alpha}}, \boldsymbol{U}=g^{\boldsymbol{\beta}}\right)$.

- Keygen $(\mathrm{msk}, \tilde{\mathbb{A}})$ : Given a non-monotonic access structure $\tilde{\mathbb{A}}$ such that we have $\tilde{\mathbb{A}}=N M(\mathbb{A})$ for some monotonic access structure $\mathbb{A}$ over a set $\mathcal{P}$ of attributes and associated with a linear secret sharing scheme $\Pi$, the algorithm applies $\Pi$ to obtain shares $\left\{\lambda_{i}\right\}$ of the master secret key $\alpha$. The party corresponding to share $\lambda_{i}$ is denoted by $\breve{x_{i}} \in \mathcal{P}$, where $x_{i}$ is the underlying attribute, and can be primed (i.e., negated) or unprimed (non-negated). For each $i$, the algorithm chooses $r_{i} \stackrel{R}{\leftarrow} \mathbb{Z}_{p}$, defines $\boldsymbol{\rho}_{i}=\left(\rho_{i, 1}, \ldots, \rho_{i, n}\right)^{\top}=\left(1, x_{i}, x_{i}^{2}, \ldots, x_{i}^{n-1}\right)^{\top}$. That is $\rho_{i, j}=x_{i}^{j-1}$. It then does as follows. 
- For each $i$ such that $\breve{x_{i}}$ is unprimed (i.e., non-negated), the algorithm computes a tuple $D_{i}=\left(D_{i, 1}^{(1)}, D_{i, 2}^{(2)}, K_{\boldsymbol{\rho}_{i}, i}^{(3)}\right) \in \mathbb{G}^{n+1}$ where the first two elements are $\left(D_{i, 1}^{(1)}, D_{i, 2}^{(1)}\right)=\left(g^{\lambda_{i}} \cdot u_{0}^{r_{i}}, g^{r_{i}}\right)$ and

$K_{\boldsymbol{\rho}_{i}, i}^{(1)}=\left(K_{i, 2}^{(1)}, \ldots, K_{i, n}^{(1)}\right)=\left(\left(u_{1}^{-\frac{\rho_{i, 2}}{\rho_{i, 1}}} \cdot u_{2}\right)^{r_{i}}, \ldots,\left(u_{1}^{-\frac{\rho_{i, n}}{\rho_{i, 1}}} \cdot u_{n}\right)^{r_{i}}\right)=g^{r_{i} \cdot M_{\boldsymbol{\rho}_{i}}^{\top} \boldsymbol{\beta}}$,

where $M_{\boldsymbol{\rho}_{i}} \in\left(\mathbb{Z}_{p}\right)^{n \times(n-1)}$ is the matrix $M_{\boldsymbol{\rho}_{i}}=\left(\begin{array}{c}-\frac{\rho_{i, 2}}{\rho_{i, 1}}-\frac{\rho_{i, 3}}{\rho_{i, 1}} \cdots-\frac{\rho_{i, n}}{\rho_{i, 1}} \\ I_{n-1}\end{array}\right)$.

- For each $i$ such that $\breve{x_{i}}$ is primed (i.e., negated), one computes a tuple $D_{i}=\left(D_{i, 1}^{(2)}, D_{i, 2}^{(2)}, K_{\boldsymbol{\rho}_{i}, i}^{(2)}\right) \in \mathbb{G}^{n+1}$ where $\left(D_{i, 1}^{(2)}, D_{i, 2}^{(2)}\right)=\left(g^{\lambda_{i}} \cdot h_{1}^{r_{i}}, g^{r_{i}}\right)$ and

$$
K_{\boldsymbol{\rho}_{i}, i}^{(2)}=\left(K_{i, 2}^{(2)}, \ldots, K_{i, n}^{(2)}\right)=\left(\left(h_{1}^{-\frac{\rho_{i, 2}}{\rho_{i, 1}}} \cdot h_{2}\right)^{r_{i}}, \ldots,\left(h_{1}^{-\frac{\rho_{i, n}}{\rho_{i, 1}}} \cdot h_{n}\right)^{r_{i}}\right)=g^{r_{i} \cdot M_{\boldsymbol{\rho}_{i}}^{\top} \boldsymbol{\alpha}} .
$$

The private key is $\mathbf{s k}_{\tilde{\mathbb{A}}}=\left\{D_{i}\right\}_{\breve{x_{i}} \in \mathcal{P}} \in \mathbb{G}^{\ell \times(n+1)}$.

- Encrypt (mpk, M, $\omega)$ : To encrypt $\mathrm{M}$ for a set $\omega$ (with $|\omega|<n$ ), it first defines $\boldsymbol{Y}=\left(y_{1}, \ldots, y_{n}\right)^{\top}$ as the vector whose first $q+1$ coordinates are the coefficients of the polynomial $P_{\omega}[Z]=\sum_{i=1}^{q+1} y_{i} Z^{i-1}=\prod_{j \in \omega}(Z-j)$. If $q+1<n$, set $y_{j}=0$ for $q+2 \leq j \leq n$. Then it picks $s \stackrel{R}{\leftarrow} \mathbb{Z}_{p}$ and computes

$$
C=\left(C_{0}, C_{1}, C_{2}, C_{3}\right)=\left(\mathrm{M} \cdot e(g, g)^{\alpha \cdot s}, g^{s},\left(u_{0} \cdot \prod_{i=1}^{n} u_{i}^{y_{i}}\right)^{s},\left(\prod_{i=1}^{n} h_{i}^{y_{i}}\right)^{s}\right) .
$$

- Decrypt $\left(\mathrm{mpk}^{\mathrm{s}} \mathrm{sk}_{\tilde{\mathbb{A}}}, \tilde{\mathbb{A}}, C, \omega\right)$ : It parses $C$ as $\left(C_{0}, C_{1}, C_{2}, C_{3}\right)$ and the private key $\mathrm{sk}_{\tilde{\mathbb{A}}}$ as $\mathrm{sk}_{\tilde{\mathbb{A}}}=\left\{D_{i}\right\}_{\breve{x_{i}} \in \mathcal{P}} \in \mathbb{G}^{\ell \times(n+1)}$. The algorithm outputs $\perp$ if $\omega \notin \tilde{\mathbb{A}}$. Otherwise, since $\tilde{\mathbb{A}}=N M(\mathbb{A})$ for some access structure $\mathbb{A}$ associated with a linear secret sharing scheme $\Pi$, we have $\omega^{\prime}=N(\omega) \in \mathbb{A}$ and we let $I=\left\{i: \breve{x_{i}} \in \omega^{\prime}\right\}$. Since $\omega^{\prime}$ is authorized in $\mathbb{A}$, the receiver can efficiently compute coefficients $\left\{\mu_{i}\right\}_{i \in I}$ such that $\sum_{i \in I} \mu_{i} \lambda_{i}=\alpha$ (although the shares are not known to the receiver). Let $\boldsymbol{Y}=\left(y_{1}, \ldots, y_{n}\right)^{\top}$ be the vector containing the coefficients of the polynomial $P_{\omega}[Z]=\prod_{j \in \omega}(Z-j)=\sum_{i=1}^{q+1} y_{i} Z^{i-1}$.

- For every positive attribute $\breve{x}_{i} \in \omega^{\prime}$ (for which $x_{i} \in \omega$ ), the decryption procedure computes $\tilde{D}_{i, 1}^{(1)}=D_{i, 1}^{(1)} \cdot \prod_{j=2}^{n} K_{i, j}^{(1)^{y_{j}}}=g^{\alpha} \cdot\left(u_{0} \cdot u_{1}^{y_{1}} \cdots u_{n}^{y_{n}}\right)^{r_{i}}$, and then $e(g, g)^{\lambda_{i} s}=e\left(C_{1}, \tilde{D}_{i, 1}^{(1)}\right) / e\left(C_{2}, D_{i, 2}^{(1)}\right)$.

- For each negated attribute $\breve{x}_{i} \in \omega^{\prime}$ (for which $\left.x_{i} \notin \omega\right)$, the receiver sets $\boldsymbol{\rho}_{i}=\left(1, x_{i}, \ldots, x_{i}^{n-1}\right)^{\top}$ and successively computes

$$
\begin{aligned}
K_{i}^{(2)} & =\prod_{j=2}^{n} K_{i, j}^{(2)^{y_{j}}}=\left(h_{1}^{-\left\langle\boldsymbol{\rho}_{i}, \boldsymbol{Y}\right\rangle / x_{1}} \cdot h_{1}^{y_{1}} \cdots h_{n}^{y_{n}}\right)^{r_{i}} \\
\tau_{i} & =\left(\frac{e\left(K_{i}^{(2)}, C_{1}\right)}{e\left(C_{3}, D_{i, 2}^{(2)}\right)}\right)^{-\frac{\rho_{i, 1}}{\left\langle\boldsymbol{\rho}_{i}, \boldsymbol{Y}\right\rangle}}=e\left(g, h_{1}\right)^{r_{i} s}
\end{aligned}
$$

and then $e(g, g)^{\lambda_{i} s}=e\left(C_{1}, D_{i, 1}^{(2)}\right)^{-1} \cdot \tau_{i}^{-1}$.

Finally, decryption computes $\mathrm{M}=C_{0} \cdot \prod_{i \in I} e(g, g)^{-\mu_{i} \lambda_{i} s}$. 
If we split $I$ into $I_{0} \cup I_{1}$, where $I_{0}$ and $I_{1}$ correspond to unprimed and primed attributes, respectively, decryption can more efficiently compute

$$
\begin{aligned}
e(g, g)^{\alpha \cdot s}=e\left(C_{1}, \prod_{i \in I_{0}} \tilde{D}_{i, 1}^{(1)^{\mu_{i}}} \cdot \prod_{i \in I_{1}}\left(D_{i, 1}^{(2)} \cdot K_{i}^{\left.\left.(2)^{\frac{\mu_{i} \cdot \rho_{i, 1}}{\left\langle\boldsymbol{\rho}_{i}, \mathbf{Y}\right\rangle}}\right)\right)}\right.\right. \\
\cdot e\left(C_{2}, \prod_{i \in I_{0}} D_{i, 2}^{(1)^{\mu_{i}}}\right) \cdot e\left(C_{3}, \prod_{i \in I_{1}} D_{i, 2}^{(2) \frac{\mu_{i} \cdot \rho_{i, 1}}{\left\langle\boldsymbol{\rho}_{i}, \boldsymbol{Y}\right\rangle}}\right),
\end{aligned}
$$

so that only three pairing evaluations are necessary.

Theorem 3. The above KP-ABE system with the maximal bound $n$ for the number of attributes per ciphertext (i.e., $|\omega|<n$ ) is selectively secure if the $n$ DBDHE assumption holds. (The proof is given in the full version of the paper).

\section{Comparisons}

Table 2 compares efficiency among available expressive KP-ABE schemes that support non-monotonic access structures. Comparisons are made in terms of ciphertext overhead, private key size as well as in the number of pairing evaluations and exponentiations (in $\mathbb{G}$ and $\mathbb{G}_{T}$ ) upon decryption.

We remark that the functionality of KP-ABE in 31] is slightly different from the original one 20. For self-containment, we re-formalize it in appendix A, where we also briefly propose a modification of KP-ABE 31] so as to have the same functionality as the original ABE. We also include this modified scheme in Table 2, Note that 31] has a unique feature of being adaptively secure.

Table 2. Efficiency of non-monotonic KP-ABE schemes

\begin{tabular}{lccccc}
\hline Schemes & \multicolumn{2}{l}{ Ciphertext overhead Private key size } & \multicolumn{3}{l}{ Decryption cost Assumption } \\
& $|\mathbb{G}|$ & $|\mathbb{G}|$ & pair. & exp. & \\
\hline OSW [28] & $O(\bar{n})$ & $O(t \cdot \log n)$ & $O(t)$ & $O(t \cdot \bar{n})$ & DBDH \\
LSW [24] & $O(\bar{n})$ & $O(t)$ & $O(t)$ & $O(t \cdot \bar{n})$ & $n$-MEBDH \\
OT [31] & $O(\bar{n} \cdot \varphi)$ & $O(t \cdot \varphi)$ & $O(t \cdot \varphi)$ & $O(t)$ & DLIN \\
OT ${ }^{\text {modified }}$ & $O(\bar{n} \cdot n)$ & $O(t \cdot n)$ & $O(t \cdot n)$ & $O(t)$ & DLIN \\
This work & 3 & $O(t \cdot n)$ & 3 & $O(t)$ & $n$-DBDHE \\
\hline
\end{tabular}

$\bar{n}=\mid$ attribute set $|=| \omega \mid$ for a ciphertext; $n=$ the maximal bound for $\bar{n}$ (i.e., $|\omega|<n) ; t=\#$ of attributes in an access structure for a key; $\varphi=$ maximum size for repetition of attribute label per key (only for the KP-ABE with labeling, formalized in appendix A .

$\ddagger$ pair., exp. shows \# of pairing and exponentiation computation (in $\mathbb{G}$ or $\mathbb{G}_{T}$ ), respectively.

\section{Concluding Remarks}

This paper presented the first results for expressive KP-ABE schemes with constant-size ciphertexts. In the future, it will be interesting to see if shorter private keys can be obtained without affecting the expressivity or the size of ciphertexts and to construct adaptively secure such schemes. Another challenging problem is to achieve similar results in the expressive ciphertext-policy setting. 


\section{References}

1. Abdalla, M., Kiltz, E., Neven, G.: Generalized Key Delegation for Hierarchical Identity-Based Encryption. In: Biskup, J., López, J. (eds.) ESORICS 2007. LNCS, vol. 4734, pp. 139-154. Springer, Heidelberg (2007)

2. Attrapadung, N., Imai, H.: Dual-Policy Attribute Based Encryption. In: Abdalla, M., Pointcheval, D., Fouque, P.-A., Vergnaud, D. (eds.) ACNS 2009. LNCS, vol. 5536, pp. 168-185. Springer, Heidelberg (2009)

3. Attrapadung, N., Imai, H.: Conjunctive Broadcast and Attribute-Based Encryption. In: Shacham, H., Waters, B. (eds.) Pairing 2009. LNCS, vol. 5671, pp. 248-265. Springer, Heidelberg (2009)

4. Attrapadung, N., Libert, B.: Functional Encryption for Inner Product: Achieving Constant-Size Ciphertexts with Adaptive Security or Support for Negation. In: Nguyen, P.Q., Pointcheval, D. (eds.) PKC 2010. LNCS, vol. 6056, pp. 384-402. Springer, Heidelberg (2010)

5. Bethencourt, J., Sahai, A., Waters, B.: Ciphertext-Policy Attribute-Based Encryption. IEEE Symposium on Security and Privacy (S\&P), pp. 321-334 (2007)

6. Boneh, D., Boyen, X.: Efficient Selective-ID Secure Identity-Based Encryption Without Random Oracles. In: Cachin, C., Camenisch, J.L. (eds.) EUROCRYPT 2004. LNCS, vol. 3027, pp. 223-238. Springer, Heidelberg (2004)

7. Boneh, D., Boyen, X., Goh, E.-J.: Hierarchical Identity Based Encryption with Constant Size Ciphertext. In: Cramer, R. (ed.) EUROCRYPT 2005. LNCS, vol. 3494, pp. 440-456. Springer, Heidelberg (2005)

8. Boneh, D., Franklin, M.: Identity-Based Encryption from the Weil Pairing. Journal of Computing 32(3), 586-615 (2003); Boneh, D., Franklin, M.: Identity-Based Encryption from the Weil Pairing. In: Kilian, J. (ed.) CRYPTO 2001. LNCS, vol. 2139, pp. 213-229. Springer, Heidelberg (2001)

9. Boneh, D., Gentry, C., Waters, B.: Collusion Resistant Broadcast Encryption with Short Ciphertexts and Private Keys. In: Shoup, V. (ed.) CRYPTO 2005. LNCS, vol. 3621, pp. 258-275. Springer, Heidelberg (2005)

10. Boneh, D., Hamburg, M.: Generalized Identity Based and Broadcast Encryption Schemes. In: Pieprzyk, J. (ed.) ASIACRYPT 2008. LNCS, vol. 5350, pp. 455-470. Springer, Heidelberg (2008)

11. Boneh, D., Sahai, A., Waters, B.: Functional Encryption: Definitions and Challenges. In: TCC 2011 (2011) (to appear)

12. Boyen, X.: General Ad Hoc Encryption from Exponent Inversion IBE. In: Naor, M. (ed.) EUROCRYPT 2007. LNCS, vol. 4515, pp. 394-411. Springer, Heidelberg (2007)

13. Canetti, R., Halevi, S., Katz, J.: A Forward-Secure Public-Key Encryption Scheme. In: Biham, E. (ed.) EUROCRYPT 2003. LNCS, vol. 2656, pp. 254-271. Springer, Heidelberg (2003)

14. Canetti, R., Halevi, S., Katz, J.: Chosen-Ciphertext Security from Identity-Based Encryption. In: Cachin, C., Camenisch, J.L. (eds.) EUROCRYPT 2004. LNCS, vol. 3027, pp. 207-222. Springer, Heidelberg (2004)

15. Chase, M.: Multi-authority Attribute Based Encryption. In: Vadhan, S.P. (ed.) TCC 2007. LNCS, vol. 4392, pp. 515-534. Springer, Heidelberg (2007)

16. Chase, M., Chow, S.: Improving privacy and security in multi-authority attributebased encryption. In: ACM-CCS 2009, pp. 121-130 (2009)

17. Cheon, J.-H.: Security Analysis of the Strong Diffie-Hellman Problem. In: Vaudenay, S. (ed.) EUROCRYPT 2006. LNCS, vol. 4004, pp. 1-11. Springer, Heidelberg (2006) 
18. Cheung, L., Newport, C.: Provably secure ciphertext policy ABE. In: ACM-CCS 2007, pp. 456-465 (2007)

19. Emura, K., Miyaji, A., Nomura, A., Omote, K., Soshi, M.: A Ciphertext-Policy Attribute-Based Encryption Scheme with Constant Ciphertext Length. In: Bao, F., Li, H., Wang, G. (eds.) ISPEC 2009. LNCS, vol. 5451, pp. 13-23. Springer, Heidelberg (2009)

20. Goyal, V., Pandey, O., Sahai, A., Waters, B.: Attribute-based encryption for finegrained access control of encrypted data. In: ACM CCS 2006, pp. 89-98 (2006)

21. Goyal, V., Jain, A., Pandey, O., Sahai, A.: Bounded Ciphertext Policy Attribute Based Encryption. In: Aceto, L., Damgård, I., Goldberg, L.A., Halldórsson, M.M., Ingólfsdóttir, A., Walukiewicz, I. (eds.) ICALP 2008, Part II. LNCS, vol. 5126, pp. 579-591. Springer, Heidelberg (2008)

22. Herranz, J., Laguillaumie, F., Ràfols, C.: Constant Size Ciphertexts in Threshold Attribute-Based Encryption. In: Nguyen, P.Q., Pointcheval, D. (eds.) PKC 2010. LNCS, vol. 6056, pp. 19-34. Springer, Heidelberg (2010)

23. Katz, J., Sahai, A., Waters, B.: Predicate Encryption Supporting Disjunctions, Polynomial Equations, and Inner Products. In: Smart, N.P. (ed.) EUROCRYPT 2008. LNCS, vol. 4965, pp. 146-162. Springer, Heidelberg (2008)

24. Lewko, A., Sahai, A., Waters, B.: Revocation Systems with Very Small Private Keys. In: IEEE Symposium on Security and Privacy (S\&P) (2010)

25. Lewko, A., Okamoto, T., Sahai, A., Takashima, K., Waters, B.: Fully Secure Functional Encryption: Attribute-Based Encryption and (Hierarchical) Inner Product Encryption. In: Gilbert, H. (ed.) EUROCRYPT 2010. LNCS, vol. 6110, pp. 62-91. Springer, Heidelberg (2010)

26. Naor, M.: On Cryptographic Assumptions and Challenges. In: Boneh, D. (ed.) CRYPTO 2003. LNCS, vol. 2729, pp. 96-109. Springer, Heidelberg (2003)

27. Naor, M., Pinkas, B.: Efficient Trace and Revoke Schemes. In: Frankel, Y. (ed.) FC 2000. LNCS, vol. 1962, pp. 1-20. Springer, Heidelberg (2001)

28. Ostrovsky, R., Sahai, A., Waters, B.: Attribute-based encryption with nonmonotonic access structures. In: ACM CCS 2007, pp. 195-203 (2007)

29. Sahai, A., Waters, B.: Fuzzy Identity-Based Encryption. In: Cramer, R. (ed.) EUROCRYPT 2005. LNCS, vol. 3494, pp. 457-473. Springer, Heidelberg (2005)

30. Shamir, A.: Identity-Based Cryptosystems and Signature Schemes. In: Blakely, G.R., Chaum, D. (eds.) CRYPTO 1984. LNCS, vol. 196, pp. 47-53. Springer, Heidelberg (1985)

31. Okamoto, T., Takashima, K.: Fully secure functional encryption with general relations from the decisional linear assumption. In: Rabin, T. (ed.) CRYPTO 2010. LNCS, vol. 6223, pp. 191-208. Springer, Heidelberg (2010)

32. Waters, B.: Ciphertext-Policy Attribute-Based Encryption: An Expressive, Efficient, and Provably Secure Realization. In: Catalano, D., et al. (eds.) PKC 2011. LNCS, vol. 6571, pp. 90-107. Springer, Heidelberg (2011)

33. Yamada, S., Attrapadung, N., Hanaoka, G., Kunihiro, N.: Generic Constructions for Chosen-Ciphertext Secure Attribute Based Encryption. In: Catalano, D., et al. (eds.) PKC 2011. LNCS, vol. 6571, pp. 90-107. Springer, Heidelberg (2011)

\section{A Variant: KP-ABE with Labeling}

We re-formalize the $\mathrm{KP}-\mathrm{ABE}$ definition of 31] in our context as follows. Intuitively, the difference from normal $\mathrm{KP}-\mathrm{ABE}$ is that an attribute is required to 
be labeled with a number $j \in[1, n]$ and that each attribute in the set associated to a ciphertext is required to be labeled uniquely, namely 1 to $n$. The scheme of 31] further restricts the maximum repetition allowed for labels in one policy, which we denote by $\varphi$ in Table 2 .

Definition 8 (KP-ABE with labeling). Let $U$ be an attribute space and let an integer $n \in \mathbb{N}$. Define $U^{\prime}=\{(j, u) \mid j \in[1, n], u \in U\}$. Define the ciphertext index domain as $\Sigma_{e}^{\mathrm{KP}^{\prime}}=\left\{\left\{\left(1, u_{1}\right), \ldots,\left(n, u_{n}\right)\right\} \mid u_{1}, \ldots, u_{n} \in U\right\}$. A KP-ABE with labeling for a collection $\mathcal{A S}^{\prime}$ of access structures over $U^{\prime}$ is a functional encryption for $R^{\mathrm{KP}^{\prime}}: \mathcal{A S}^{\prime} \times \Sigma_{e}^{\mathrm{KP}^{\prime}} \rightarrow\{0,1\}$ defined by $R^{\mathrm{KP}^{\prime}}(\mathbb{A}, \omega)=1$ iff $\omega \in \mathbb{A}$ $\left(\right.$ for $\left.\omega \in \Sigma_{e}^{K \mathrm{P}^{\prime}}, \mathbb{A} \in \mathcal{A} \mathcal{S}^{\prime}\right)$.

We observe that KP-ABE with large universe $U=\{0,1\}^{*}$, e.g., 20, 28, and ours, implies KP-ABE with labeling. This is since $U^{\prime} \subset U, \Sigma_{e}^{\mathrm{KP}} \subset \Sigma_{e}^{\mathrm{KP}}, \Sigma_{k}^{\mathrm{K} \mathrm{P}^{\prime}} \subset$ $\Sigma_{k}^{\mathrm{KP}}$, and $R^{\mathrm{KP}} \Leftrightarrow R^{\mathrm{KP}}$ holds and the implication comes from the embedding lemma [10,4. To the best of our knowledge, the converse is yet known to hold.

We now briefly propose a KP-ABE that conforms with the normal definition by modifying [31. We construct by instantiating the general KP-FE scheme of [31] with $d=1$, and with the inner product relation being instantiated to IBBE, similarly as we did in section 3.3. and setting the bound $\varphi=n$. 